\title{
ABSTRACTS
}

\section{CAGP-CCSMH Annual Scientific Meeting: Book of Abstracts}

\author{
GERIATRIC PSYCHIATRY IN CHALLENGING TIMES: \\ EMBRACING OPPORTUNITIES FOR ADVOCACY, INCLUSION AND INNOVATION: \\ OCTOBER 29-30, 2021
}

https:10.5770/cgj.25.595

\section{Challenges to Maintaining Wellness in Challenging Times: How to Cope}

\section{Ken Schwartz, Robert Madan}

Background: Who helps the caregiver? We are all vulnerable to stress and even the strongest and healthiest of us can struggle to maintain wellness in unsupportive healthcare and societal environments. How can healthcare providers advocate for more support for both our emotional and physical wellbeing and for the vulnerable elderly living in community and long- term care homes.

Methods: A tripartite model of coping is presented and focuses on the importance of sharing emotions, taking action to solve problems and finding meaning in our work while encountering personal,work and societal stresses exacerbated by the pandemic. The consequences of not doing so can and will lead to distress, possible burnout and poorer patient outcomes. Vignettes relating to challenging clinical and personal work situations will be presented and invited from participants while addressing equity, diversity and inclusion

Results: Evidence showing connections between wellness, patient outcomes, improved team functioning and quality of life is presented. Unfortunately, evidence shows that burnout amongst healthcare providers is common, with few taking action and seeking help. Instead, feelings are kept to oneself only to remain "the elephant in the room." This workshop will model effective ways of identifying the need for action and steps to achieving healthier work-life balance.

Conclusions: It has been said that without mental health there is no health and that the secret of care for patients is caring for the patient and healthcare providers. Using a didactic and experiential format participants are invited to share their work and personal struggles in maintaining wellness and resilience. In doing so, we have attempted to help answer the question of who helps the caregiver in these challenging times.

\section{Implementing a Care Pathway for Detecting and Treating MCI, Depression andAnxiety in Primary Care}

\begin{abstract}
Nick Kates
Background: With the high prevalence and burden of anxiety depression and mild cognitive impairment in later life, earlier detection and treatment could both improve well being and modify the risk for Alzheimer's disease. As most individuals with these conditions present in primary care, rarely reaching specialised services, we examined whether collaborative approaches between primary care and mental health care clinicians could support the introduction of integrated care pathways to assist with recognition, assessment, and standardize care.
\end{abstract}

Methods: We introduced an integrated pathway to diagnose and manage anxiety, depression and MCI in 5 primary care practices in Ontario. At the same time, we tracked changes made by the practices to incorporate the pathway, held focus groups with participating practices to learn about their experiences and conducted a chart review to identify possible changes in detection rates. The study took place over a two-year period.

Results: Introducing the pathway was feasible and resulted in a reduction in symptoms and improvement in quality of life. It led to better access to recommended treatments and increased detection rates for anxiety, depression and MCI although this was not sustained once the support from the study was withdrawn. Factors facilitating its introduction included an identified lead for the project in the practice, physician engagement, and limiting the demands made of physicians.

Conclusions: The pathway is effective in improving detection rates and access to care. Lessons learnt from the focus groups and process evaluation identified key steps to assist with its spread to other practices. 
Anxiety

\section{Nick Kates, Tarek Rajji}

Background: With the high prevalence and burden of anxiety depression and mild cognitive impairment in later life, earlier detection and treatment could both improve well being and modify the risk for Alzheimer's disease. As most individuals with these conditions present in primary care, rarely reaching specialised services, we examined whether collaborative approaches between primary care and mental health care clinicians could support the introduction of integrated care pathways to assist with recognition, assessment, and standardize care.

Methods: We introduced an integrated pathway to diagnose and manage anxiety, depression and MCI in 5 primary care practices in Ontario. At the same time, we tracked changes made by the practices to incorporate the pathway, held focus groups with participating practices to learn about their experiences and conducted a chart review to identify possible changes in detection rates. The study took place over a twoyear period.

Results: Introducing the pathway was feasible and resulted in a reduction in symptoms and improvement in quality of life. It led to better access to recommended treatments and increased detection rates for anxiety, depression and MCI although this was not sustained once the support from the study was withdrawn. Factors facilitating its introduction included an identified lead for the project in the practice, physician engagement, and limiting the demands made of physicians.

Conclusions: The pathway is effective in improving detection rates and access to care. Lessons learnt from the focus groups and process evaluation identified key steps to assist with its spread to other practices.

\section{Tarek Rajji}

Background: An integrated care pathway had already been developed at CAMH and the goal was to look at its effectiveness when introduced into a primary care setting. We wanted to look at three measures - a) the detection rates for depression anxiety and MCI and primary care; b) the time to treatment for individuals identified with one of these problems, and c) outcomes for individuals in the integrated pathway methods.

Methods: The main assessment tools were an PHQ9 GAD 7 MOCA and QOL 69 participants were enrolled in the ICP arm and 76 in the TAU arm, in 5 different practices. The ICP included medication, patient education and social activation. Follow-up assessments were conducted at baseline and then every 6 months for up to 2 years.

Results: Participants did not differ in age, gender, marital status, education, or employment status. Participants assigned to ICP experienced more persistent improvement on measures of depression, anxiety and quality of life than those assigned to TAU. They were also more likely to access the right treatment and faster than those in TAU. With active screening, detection rates of anxiety, depression, or MCI increased 5-fold compared to before starting the study. However, rates of detection went back to before the study after the withdrawal of active screening.

Conclusions: An integrated care pathway can be adapted and integrated successfully into primary care and appears to play an important role in enhancing care, improving access and time to treatment, and outcomes for individuals with depression anxiety and mild cognitive impairment.

\section{Integrating a Care Pathway Within Primary Care: Adjustments Required and Lessonsfor Spread}

\section{Nick Kates}

Background: While the integrated care pathways improved outcomes and access to care, we also wanted to examine what changes practices needed to make to support its successful implementation, and prepare for its possible spread to other practices.

Methods: As the ICP was introduced, we monitored changes that practices reported and problems that arose. We held six monthly focus groups with family physicians to get their feedback on the model and the changes they had had to make to learn how to adapt it to other practices.

Results: The ICP was seen positively by most family physicians despite the increasing time demands. Physicians emphasised the need to keep this to a minimum when introducing a new program. A dedicated person in the practice to coordinate the initiative and screening is important and the system changes are required to accommodate an ICP. The algorithm may need to be adapted for individual practices and outcomes were positively affected by greater physician buy in and engagement. A shorter time to treatment indicated that physicians were using the algorithm.

Conclusions: Integrating an ICP in primary care, requires a minimum of time demands of family physicians and wider system changes to support its introduction. There may be benefits in providing materials electronically, both for training physicians and for patient education. Buy in from the physicians is probably the most important factor, accompanied by information about the importance of early recognition of these problems and about seniors' mental health in primary care in general.

\section{Facilitating Person-Centred Transitions Across Sectors Using 'My Transitional Care PlanDuring the COVID-19 Pandemic'}

\section{Katelynn Aelick, Monica Bretzlaff, Debbie Hewitt Colborne, Teresa Judd, Jillian McConnell, Jacquie Seguin, Kylie Turcotte}

Background: Following the initiation of the pandemic, hospitals worked diligently to expand their capacity. Individuals no longer needing acute care services were moved into new environments including long term care homes and off-site 
temporary housing. Many of these individuals were older adults living with dementia and other complex health conditions, making it essential to develop a comprehensive transition plan that took into account the extenuating circumstances of COVID-19.

Methods: 'My Transitional Care Plan during the Covid-19 Pandemic' (MTCPC19) was adapted from 'My Transitional Care Plan' - a tool originally developed in Northeastern Ontario. Its adaptation took place over several months during which key themes were identified by health care providers working in multiple sectors across Ontario. Key themes included the person's typical daily routines, common responsive behaviours, approaches to support them in the pandemic context, ways to keep them socially connected and other elements to support the diverse individuals in need of transitional support.

Results: MTCPC19 was released publicly for trial in October 2020. Feedback collected during the trial informed the development of a final version which was released in Spring 2021. Alongside the release of the bilingual tool, guidelines to support its use were created alongside a completed example.

Conclusions: MTCPC19 was created to optimize transitions from one environment to another during the COVID-19 Pandemic. The use of this tool can equip health care providers with a practical resource to enhance communication and collaboration while upholding the continuity of care needed to support a safe and sustainable transition.

\section{The Role of the Psychiatric Clinical Nurse Specialist in Improving Delirium/DementiaManagement and Building Mental Health Nursing Competencies in Acute Care Settings: A One-Year Pilot}

\section{Linda Liu}

Background: Mental health nursing competencies are often considered a specialized clinical skillset. However, as nurses in acute care settings care for an increasing aging population, they find themselves ill-equipped to therapeutically and safely manage neuropsychiatric symptoms related to dementia and/ or delirium. This presentation will describe the implementation of a one-year pilot Psychiatric clinical nurse specialist (CNS) role within the General Internal Medicine and Surgery programs at an academic tertiary care hospital, and role integration into a Geriatric C-L psychiatry service.

Methods: Clinical indicators were established to measure the impact of the Psychiatric CNS role on length of stay, restraint use and constant observation resources. Surveys were also collected near the end of the pilot period to examine clinician perspectives regarding the impact of the Psychiatric CNS role on clinical practice, and clinicians' skill and confidence to manage responsive behaviours.

Results: The Psychiatric CNS was found to be an effective role in developing clinician capacity in the use of non-pharmacological strategies to address dementia and/or delirium responsive behaviours.
Conclusions: An interdisciplinary geriatric psychiatry team model provides broadened skillsets to effectively manage neuropsychiatric symptoms related to dementia and/or delirium. The addition of a Psychiatric clinical nurse specialist role can build clinician capacity in non-pharmacological strategies to address dementia and/or delirium responsive behaviours.

\section{Behavioural Supports in Acute Care: Collective Voices Leading to KeyRecommendations}

\author{
Debbie Hewitt Colborne, Natasha Fortin, \\ Jillian McConnell, Nancy Lesiuk, Terri Glover, \\ Jennifer Koop, Teresa Judd
}

Background: The Behavioural Supports Ontario (BSO) Acute Care Collaborative launched in late 2019 to bring together health care professionals, leaders and individuals with lived experience in order to identify and spread best/emerging practices in providing person and family-partnered behavioural supports within acute care settings. Older adults often require acute care visits and necessitate unique care approaches.

Methods: In early 2020, the BSO Acute Care Collaborative released Behavioural Supports in Acute Care - Current Practices and Opportunities for Growth; a survey intended to provide a snapshot of current practices, gaps and opportunities for growth within acute care hospitals in supporting older adults living with, or at risk of, responsive behaviours/ personal expressions associated with dementia, complex mental health, substance use and/or other neurological conditions. The survey results were collated and summarized into a report, which was released in February of 2021.

Results: The survey collected over 250 responses from across Ontario. Respondents were primarily acute care clinicians, BSO clinicians and acute care and BSO leaders. All survey respondents indicated a need to further support older adults with responsive behaviours/personal expressions throughout their hospital visit/stay. Review and analysis of the survey results led to the identification of key themes and recommendations featured in the report.

Conclusions: These survey results will be used by the Collaborative to direct their collective work to identify and spread best and emerging practices in providing inclusive, person and family-partnered behavioural support within acute care hospitals. Clinical teams and organizations are encouraged to consider their role in moving these key recommendations forward.

\section{A Comparison of Frameworks for Behavioural Intervention in NeuropsychiatricSymptoms of Dementia: Using the Tools in the Toolbox}

\section{Robert Madan, Kenneth Schwartz}

Background: Neuropsychiatric Symptoms in dementia (NPS) are extremely common and are associated with poor outcomes and caregiver burden. A variety of frameworks and tools exist 
to assess and understand the symptoms in the clinical context, and to plan and operationalize interventions. These frameworks and tools are not mutually exclusive as they can be used flexibly together as part of a comprehensive approach to NPS.

Methods: Frameworks and tools such as P.I.E.C.E.S, D.I.C.E, ABC, Gentle Persuasive Approach, and the Baycrest QuickResponse Caregiver Tool ${ }^{\mathrm{TM}}$ will be described and the evidence will be reviewed. Participants will compare and contrast the tools and apply them to a case to demonstrate that they can work synergistically to assist the caregiver and care team to inform, plan, operationalize, and assess a comprehensive behavioural intervention.

Results: Care teams can use the frameworks and tools flexibly either concurrently or at different times in the episode of care of persons with dementia and NPS. These tools must address the diversity and personal background of the person with dementia and caregivers.

Conclusions: NPS is common in dementia and present a significant challenge the caregivers, health care providers, and the health care system. There are various tools in the NPS toolbox and there is no "one perfect tool for the job." The frameworks and tools complement each other and can be used flexibly.

\section{A New Model for Assertive Community Treatment (ACT): Geriatric ACT Team}

\section{Sarah Colman}

Summary: In the era of deinstitutionalization, assertive community treatment (ACT) was developed as an alternative to hospitalization for patients with severe and chronic mental illness. Studies around the world, including Canada, have shown that ACT teams reduce hospitalizations and improve quality of life for these patients. As a result of researchers' early focus on fidelity and reproducibility, the ACT model has been successfully adapted to various unique patient populations including youth, forensic and dual diagnoses. Older adults living with severe mental illness represent a particularly vulnerable and often underserved population due to comorbid medical conditions, cognitive impairment, decreased social supports, housing insecurity and reduced financial resources. As such, there has been increasing focus ondeveloping interdisciplinary community outreach for older adults. Unfortunately, in Canada, there remains significant variation in the inclusion criteria for older adults in ACT teams and few are specifically designed to care for this population. To service this gap, a novel geriatric ACT team was developed through Reconnect in Toronto, which is uniquely tailored to meet the complex needs of older adults, in part, by emphasizing the medical-psychiatric interplay.

In this symposium, we will begin by reviewing the current landscape of psycho-geriatric care within Toronto's ACT teams, including statistical data and insights from ACT team members. We will then discuss the implementation and core components of a novel geriatric ACT team in Toronto. We will emphasize key barriers and facilitators to implementation and highlight important considerations for diversity and inclusion in development of this program.

\section{Illness: Exploration of Practitioners' Perspectives}

\section{Michael Tau, Claire Stanley, Sarah Colman}

Background: As the world's population ages, it is expected that the number of older adults living with severe mental illness (SMI) will grow. Assertive community treatment (ACT) is an established model of community care that has been shown to improve outcomes for clients with SMI. Yet there is limited research about the use of ACT for older adults with SMI. This study was designed to explore ACT team members' perspectives on providing care to older adults.

Methods: Toronto ACT teams provided quantitative data regarding the number of older adults on their rosters. ACT team staff members participated in semi-structured interviews regarding their experiences caring for older clients. Interviews were analyzed using thematic analysis.

Results: In this sample, older adults comprised a significant minority of the roster of ACT teams, with one third of clients over the age of 55 and $8.4 \%$ above 65 . Some teams had upper age limits for eligibility. Several themes emerged from our qualitative data; overarching categories were physical health, supports and services, geriatric "expertise," and the tension between team versality and strain. Participants varied in whether they felt empowered and mandated to care for older clients, versus burdened by staffing shortages, finite health system resources, and lack of expertise.

Conclusions: ACT teams were challenged by the complexity of their older clients, who comprise a significant minority of team rosters. Potential models to address some of these issues might include dedicated psychogeriatric ACT teams, integration of psychogeriatric specialists into existing teams, or improved access to geriatric consultation.

\section{Facilitators}

\section{Claire Stanley, Michael Tau, Sarah Colman}

Background: Although ACT has been shown to be a reproducible and adaptable model, to date, there has only been one randomized controlled trial studying a geriatric ACT team. The promising results of this study, in conjunction with the growing body of literature reinforcing the importance of geriatric outreach, supports the development of a geriatric ACT team. This segment will examine the implementation of Reconnect's novel geriatric ACT team in Toronto, which was initiated in February 2020.

Methods: Core components of the geriatric ACT team and its fidelity to the traditional ACT model will be discussed. Using qualitative analysis, we will highlight key barriers and facilitators identified in the implementation of this program. Given the 
inherent vulnerability of this marginalized population, considerations for diversity and inclusion will be discussed throughout.

Results: Key distinguishing features from traditional ACT will be highlighted. Reconnect's geriatric ACT team was designed to meet the complex needs of older adults and embrace the medical-psychiatric interplay prevalent in this population. In addition to psycho-geriatric care, the team focuses on addressing "geriatric giants" such as mobility, cognitive impairment and polypharmacy. This includes integrated assessments by geriatricians and mobility/falls assessments by occupational therapy. Quantitative and qualitative data, collected over an eighteen-month period, will be presented to highlight demographics and barriers and facilitators to implementation.

Conclusions: Studying the implementation of a novel approach to care is essential to facilitate future research and reproducibility of this model. We will conclude with a discussion about research directions and future landscape for psycho-geriatric outreach.

\section{Long-Term Care During COVID-19}

\section{Dallas Seitz, Claire Checkland, Sophiya Benjamin, Marie-Andree Bruneau, Antonia Cappella, Beverley Cassidy, David Conn, Cindy Grief, Alvin Keng, et al.}

Background: COVID-19 has disproportionately impacted older adults in long-term care (LTC) facilities in Canada. Addressing the mental health needs of older adults in longterm care during COVID-19 is important and has not been adequately addressed in existing long-term care COVID-19 policy documents.

Methods: The Canadian Academy of Geriatric Psychiatry (CAGP) and Canadian Coalition for Seniors Mental Health (CCMSH) established a working group to develop position statements on mental health care for older adults in LTC settings during COVID-19. Eight statements were developed by the working group.

Results: The position statements reflected several key messages. Mental health care is an essential medical service that must be maintained during any pandemic. Older adults living in LTC facilities have the right to mental health, medical care and social services, regardless of their age, the presence of dementia, or a diagnosis of other mental health problems and illnesses. LTC facilities must be provided with the technology and resources necessary for provision of essential virtual medical services and for the maintenance of family and social connections when in-person visits are restricted. Infection control measures intended to reduce the spread of infectious diseases must also balance the impact of these measures on the quality of life and dignity of LTC residents. Supporting the mental health of LTC staff is critical to effectively managing pandemics in LTC.

Conclusions: The CAGP/CCSMH position statement can be used by clinicians to advocate for needed improvements to mental health care in long-term care during COVID-19 and in the future.

\section{Toolkit}

\section{Andrea Iaboni, Alisa Grigorovich, Pia Kontoa, Arlene Astell, Josephine McMurray, Charlene Chu, Kevin Rodrigues, Claudia Barned, Dementia Isolation Toolkit Team}

Background: The infection control measures used to prevent the spread of COVID-19 have had a severe impact on the well-being of long-term care (LTC) residents and moral distress for staff. LTC staff have been asked to adhere to professional, institutional, and public health guidelines, while also minimizing the consequences for residents and supporting person- centred care. In LTC, moral distress can occur when staff perceive that the ethically appropriate action is at odds with the directives they receive. There is thus a need in the LTC environment for resources that support the moral resilience of the staff.

Methods: The research team, in collaboration with LTC stakeholders, developed the Dementia Isolation Toolkit (DIT). The DIT helps to build moral resilience in LTC staff by providing an ethical decision-making framework and tools to support person- centred care-planning in a pandemic. We have also conducted a survey of over 200 LTC staff in Ontario to understand the impact and use of the DIT.

Results: The DIT has been downloaded more than 6500 times since it was published (bit.ly/dementiatoolkit) and has been disseminated internationally. However, there is a need for additional supports for ethical decision-making in the context of a pandemic in the LTC setting.

Conclusions: The pandemic has highlighted the need for the development and implementation of tools to support personcentred care planning to reduce the harms associated with isolation, as well as the moral resilience of LTC staff as they navigate ethical dilemmas during COVID-19.

\section{Vanessa Thoo, Leslie Giddens-Zuker}

Background: The COVID-19 pandemic has had a devastating impact, particularly within the LTC setting, with much discussion regarding the deaths occurring in LTC homes and impact of the quarantine on responsive behaviors; however, there has been little information regarding BPSD in patients with dementia who are COVID-recovered. Even in the absence of COVID- associated delirium, there have been several cases demonstrating an increase in BPSD post-recovery.

Methods: A literature review was conducted with a focus on dementia and neuropsychiatric symptoms post-COVID-19 infection in the LTC setting. We will discuss an interdisciplinary model of geriatric mental health outreach (GMHOT) through case presentations, with emphasis on the cultural complexity of the LTC population in Toronto. 


\section{CAGP-CCSMH ANNUAL SCIENTIFIC MEETING: BOOK OF ABSTRACTS}

Results: With the COVID-19 pandemic, the assessment and management of responsive behaviours and other neuropsychiatric symptoms in the LTC setting has been complex, with virtual assessments adding another barrier. In particular, working with non-English speaking residents in Toronto homes as they experience these post-COVID issues has been of particular challenge. Without their families being able to visit, these residents have minimal social contact, especially in the absence of staff who speak their language, and virtual assessments with interpreters remain less than optimal.

Conclusions: While the evidence in limited due to the rapidly evolving nature of the SARS-Cov-2 virus, it appears that persons with advanced dementia may have persistent worsening of their neuropsychiatric symptoms after experiencing COVID. The GMHOT team can best support these patients through a combination of pharmacological and non-pharmacological interventions in managing these challenging symptoms.

\section{Sophiya Benjamin, Joanne Ho, Julie Carthew, Lindsay Cox, Rymon Rofaiel}

Background: Older adults with severe and persistent mental illnesses are a vulnerable population. Often precariously housed, with little family support, these individuals are also less likely to receive services and medications for medical comorbidities. Traditional adult mental health models may struggle to support this aging cohort. In addition to these existing pressures, the COVID 19 pandemic has exacerbated these inequities. However, the same pandemic has also acted as a catalyst and accelerated the uptake of virtual care.

Methods: Many community practitioners have reached out across geographical regions and created new teams to support vulnerable patients. As we do so, we are learning the strengths and vulnerabilities of virtual care models. Becoming aware of this can help refine these new practices.

Results: In this workshop, Ms. Julie Carthew NP will present the referrer's perspectives from her geriatric outreach work and illustrate how to make such alliances, work with remote teams as well as what the most helpful components of remote consults are. Dr. Joanne Ho will present the importance of an accurate medication history in remote consultations and explain the components of a best possible medication history. Dr. Benjamin will explore the potential medico legal aspects of working as a virtual interdisciplinary team. Ms. Lindsay Cox will provide a summary of patient perspectives obtained through qualitative feedback.

Conclusions: By reviewing the asynchronous virtual consultation process from the referrer, specialist and patient perspectives, this workshop will support specialists to hone their own virtual consult practices.

\section{There Yet?}

\section{Amer Burhan}

Summary: The symposium will give update on the rationale and utility of using Artificial Intelligence to explore biometric signature for agitation in Major Neurocognitive Disorders (MNCD), a significant challenge in the care of patients at all levels.

\section{Wearable Sensor Technology - A Synthesis of the Evidence}

\section{Elena Guseva, Andrea Iaboni, Nathan Herrmann, Dallas Seitz, Amer M. Burhan, Krista Lanctot, Andrew Lim, Machelle Wilchesky}

Background: The use of objective physiological signals from wearable sensor technology (WST) offers tremendous potential towards early identification and monitoring of neuropsychiatric symptoms (NPS) in persons with dementia (PwD). A wide array of new technologies may provide solutions, especially those explicitly designed to support people with dementia and their formal and informal caregivers.

Objectives: (1) to present the evidence for the use of WST in detecting and/or monitoring each NPS domain in PWD; (2) to present the evidence for the feasibility and acceptability of WST use in this population.

Methods: We conducted a diagnostic test accuracy systematic review. A systematic literature search was carried out from inception until December 2020 was conducted using both medical and engineering library databases. To warrant inclusion, studies were required to use WST as a diagnostic tool for NPS detection or use WST for NPS monitoring. Our protocol was registered https://www.crd.york.ac.uk/prospero/ display_record.php?RecordID $=219917$

Results: A systematic literature search produced 6,112 articles. Using the PRISMA guidelines, 103 titles were retained for analysis. The majority of studies investigated sleep disturbances 43(41.75\%), agitation 30(29.12\%), wandering/ pacing $19(18.44 \%)$, and apathy $17(16.5 \%)$. Technology used included accelerometry $45(43.69 \%)$, actigraphy $37(35.92 \%)$, and electrodermal activity 5(4.85\%). In total, 24(23.3\%) studies investigated the acceptability and feasibility of these devices for PwD.

Conclusions: This will be the first comprehensive systematic review to investigate the diagnostic test accuracy of WST for the detection and monitoring for each NPS domain. If valid, feasible and acceptable, these technologies are poised to revolutionize dementia care. 


\section{Neuropsychiatric Symptoms in People with Dementia}

\section{Andrea Iaboni, Sofija Spasojevic, Kristine Newman, Lori Schindel-Martin, Bing Ye}

Background: The neuropsychiatric symptoms of dementia can include episodic agitation or aggression in response to internal or external stressors. The objective of this study was to use a multisensor wearable (accelerometer (ACC), electrodermal activity (EDA), skin temperature (TEMP), and blood volume pulse (BVP)) to detect episodes of agitation or aggression in people living with dementia.

Methods: Older adults with advanced dementia admitted to a tertiary specialized dementia unit $(\mathrm{n}=20)$ wore an Empatica E4 wristworn device during waking hours for one month. Episodes of agitation were documented by clinical staff and validated using a system of fifteen research video cameras, to label start and end times of events. Sensor data were processed in 1 minute windows, and the resulting features underwent selection before being included in machine learning random forest classifier to categorized each minute as demonstrating agitation/no agitation, using two-fold cross-validation. Personalized models using data from each individual separately were also developed.

Results: Of the 20 participants, 17 had at least one agitation event over 239 days, of which 305 events could be fully labelled using video footage. After feature reduction, 22 features were selected as most informative. Multi-sensor models performed better than single sensor models, with multi-sensor generalized models including all 22 features providing an AUC of 0.83 for classifying agitation minutes. Personalized models performed better than generalized models.

Conclusions: The use of multimodal sensors and personalized machine learning models improve the detection of episodic agitation in people with dementia over the use of single sensors.

\section{Detect Pre-agitation Signature at the Point of Care?}

\section{Aurelia Soltan, Mervin Blair, Carolyn McGregor, Amer M. Burhan}

Background: Agitation and aggression (AA) in people living with dementia (PWD) impose a tremendous burden on individuals themselves, their families, caregivers, and the healthcare systems. Early recognition of AA episodes would allow for timely deployment of preventive measures, reduce cost of care, and lower the prevalence of critical incidents in this population. Research suggests that the utilization of artificial intelligence based on wearable technology generated real-time data can provide this timely recognition if prevailing challenges with data processing and computational models' building are methodically addressed.
Methods: In this study, we will collect data from multi-model wearable devices at the point of care in an inpatient geriatric psychiatry dementia unit. An Artificial Intelligence (AI)algorithm will detect the pre-agitation biometric "signature" (i.e. autonomous nervous system's arousal manifested by higher electrodermal activity, faster heartrate, lower heart rate variability, as well as increased motor activity) of agitation in dementia patients based on data collected. The AI algorithm will incorporate temporal (time-based) analysis of physiological data, a form of big data, from patients with advanced dementia, synchronized with the recorded in parallel observational clinical notes on agitation for consistent data labeling.

Results: The biometric signature analysis, the AA predictive accuracy rates based on big data and AI techniques, as well as the preliminary feasibility data will be presented.

Conclusions: Challenges and opportunities related to reallife, point of care implementation of multi-modal wearable devices and the use of AI to establish pre-agitation biometric signature will be discussed.

\section{Anna Skosireva, Linda Gobessi, Alan Douglass}

Background: Enhanced CBT (eCBT) group for seniors is an adapted protocol to facilitate learning of CBT techniques. Tailoring psychotherapy interventions for older adults is critical due to the complexities of late-life depression and anxiety and the impact of age-related changes in cognition. eCBT has not been extensively studied but has shown positive effects, adding to the existing evidence supporting CBT in the elderly. Most studies have classified seniors into one group where effect size estimates based on a broad age category of $>65$ years may mask age-related heterogeneity and estimates of CBT benefits across this population.

Methods: Forty participants over 65 years old with depressive and/or anxiety symptoms who completed eCBT group therapy were included in the analysis. Participants were further classified into two subgroups: $<75$ years $(n=15)$ and $\geq 75$ years $(\mathrm{n}=25)$. Pre- and post-intervention self-reported outcome measures included the Beck Depression Inventory-II (BDIII), Beck Anxiety Inventory, and Perceived Quality of Life Questionnaire. Differences in main outcome measures were analyzed by the t-test and Mann-Whitney U test.

Results: Participants in the $<75$ years old subgroup demonstrated significant improvement and large effect size on all outcome measures. Individuals $\geq 75$ years had significant improvement on BDI-II with moderate effect size but no significant change on other measures.

Conclusions: Seniors $>65$ years show clear benefit from group eCBT while the benefit is more modest in the $\geq 75$ group with gains reflected solely on BDI-II scores. Further adaptation of the model may bear exploration. 


\section{Problem Solving Therapy (PST) Program for Depression}

\author{
Julia Kirkham, Dallas Seitz, \\ Zahra Goodarzi, Emily St. Denis
}

Background: Few depressed older adults receive adequate treatment, which can include medications or psychotherapy (talk therapy). Psychotherapy is often unavailable to older adults due to high cost, limited resources, and other barriers such as lack of transportation. Internet delivered psychotherapy is a more accessible option that can be as effective as face-to-face therapy.

Methods: This project will develop, implement, and evaluate a new evidence-based treatment resource for late-life depression, Ger- iPST. Ger-iPST is an 8-week online psychotherapy program with online therapist support based on Problem Solving Therapy (PST). Older adults with symptoms of depression will be recruited at two sites in Kingston, Ontario and Calgary, Alberta. Participants will complete weekly online modules and we will measure symptoms of depression before, during, and after completing the program. We will also collect information on symptoms of anxiety, quality of life, and usability. Participants will be interviewed to provide feedback on acceptability and barriers and facilitators to use of Ger-iPST.

Results: This project is in progress; the process for developing an internet-based treatment resource will be discussed, and evidence based elements of Ger- iPST that are tailored to the specific needs of older adults (such as relevant content, process adaptations, and digital usability modifications) will be described. Preliminary results will be presented.

Conclusions: Internet-delivered therapy is a rapidly expanding area of psychiatry, but very few interventions have been developed for or targeted older adults specifically. Ger-iPST project addresses an important, emerging health challenge facing older populations: the need for safe, flexible, and remotely delivered care.

\section{Resources for Caregivers of People Living with Dementia}

\section{Riley Malvern, Saskia Sivanthanan, Nathan Christie}

Background: Knowing that people have diverse learning styles and preferences for accessing information, the Alzheimer Society of Canada (ASC) is expanding the range of educational resources that we currently provide through a traditional medium (i.e., printed information sheets) to a multimedia range of knowledge transfer and exchange (KTE) tools. Beginning with a pilot project that explored the practical application of KTE to a key education resource, ASC has developed an operational process with the involvement of diverse stakeholders to help our audiences access the information they need in a manner that accommodates them.
Methods: A resource for the pilot project was chosen based on a data driven approach to assess need and impact (i.e., number of website views, downloads, printed resource orders and feedback from stakeholders). Using ASC's KTE framework and the results of an environmental scan that identified approaches used by other organizations, the team operationalized the KTE framework with a focus on: 1) audiences, 2) information channels, 3) feasibility and 4) accessibility. Alzheimer Society support staff and family caregivers helped guide the development of the tools through focus groups.

Results: An infographic and video series on practical communication tips for caregivers were created. These KTE tools will support caregivers in staying connected to the person living with dementia at all stages of the disease.

Conclusions: The process established through this project will ensure that those who turn to ASC for information can find up-to-date and evidence-based content through a variety of tools that are engaging, easy to understand and accessible.

\section{Hoarding Disorder}

\section{Amanda Canfield, Karen Rowa}

Background: There is very little research available on hoarding disorder despite it being one of the most common mental health illnesses. This presentation will explore the evolution of the diagnosis including what is currently known about its progression across the lifespan, presentation cross-culturally, and treatment options.

Methods: During this workshop, participants will be provided with background knowledge on hoarding disorder including its diagnostic criteria, prevalence, and common comorbidities. Practice cases will be used to highlight a systematic approach to determining whether individuals meet criteria for hoarding disorder. The session will also include a summary on what is currently known about the trajectory of hoarding disorder with an emphasis on its presentation in seniors and cross- culturally. Finally, a review of the literature on management strategies will be presented with a focus on both current and potential non-pharmacologic and pharmacologic interventions. Participants will be provided with the Cognitive Behavioural Therapy model for hoarding disorder and practical skills that can be utilized when treating those with this diagnosis.

Results: By the end of the workshop, participants will be more aware of hoarding disorder as a diagnosis including its progression, presentation in seniors, and current and potential management strategies.

Conclusions: Hoarding disorder is a prevalent and chronic illness yet it often goes unrecognized and untreated. This workshop will help participants become more comfortable with the diagnosis and the available management options. 


\section{Well-being in Rural Communities}

\section{Beverley Cassidy}

Summary: Up to $40 \%$ of dementias can be prevented or delayed with preventive measures (Livingston et al, 2020). Key components in brain health promotion across the lifespan include cognitive stimulation and social connection. During COVID-19, a virtual Memory Boot Camp to improve memory function skillsets was introduced in rural Nova Scotia to promote brain health and function in a community sample of older adults. The Memory Boot Camp brought evidencebased memory skills training over a 6 -week long interactive series, with quality assurance feedback to assess feasibility and acceptability of the model.

To help address the needs of community members already experiencing neurocognitive disorders, a pilot series of virtual Memory Cafes also were created. Memory Cafes are designed to enhance cognitive stimulation, social inclusion and connection as well as quality of life for community-dwelling older adults living with dementia and their families. While Memory Cafes have existed since the late 1990's, virtual Memory Cafes are new and the COVID-19 pandemic provided the opportunity to assess the feasibility, value and acceptability of virtual Memory Cafes in two rural Nova Scotia communities during Winter and Spring 2021. Quality assurance measures to assess the feasibility and value of virtual interventions for persons with dementia and caregivers will be compared with results from in-person Memory Cafes held in Winter 2020.

\section{Boot Camp}

Gail Eskes, Ryan Wilson, Beverley Cassidy, Steven Wilton, Nick Zamora, Ashley Alders

Background: Changes in subjective memory function and objective performance on episodic memory tests are common with increasing age and contribute to declines in functional independence. A number of evidence-based memory interventions based on learning strategic compensatory strategies exist, and the purpose of this project was to apply these strategies in the development of an accessible intervention to support and enhance memory function for community living seniors.

Methods: We developed Memory Boot Camp (MBC), a psychoeducation approach to building memory skills that provided didactic information on memory, together with structured teaching and practice on evidence-based memory strategies to enhance encoding and retrieval. Initial boot camps were held in a group format in the community, but the Memory Boot Camp was recently adapted during COVID-19 to an online format in 2020 in rural Nova Scotia. We used the 'flipped classroom' approach with asynchronous educational videos combined with three one-hour synchronous Zoom sessions focused on application of material. Results from this online $\mathrm{MBC}$ anonymous quality assurance evaluation are reported here.
Results: 21/40 attendees completed our post-MBC questionnaire. Improved knowledge about memory and useful memory strategies were endorsed by $95-100 \%$ of respondents. Video teaching materials were rated positively by $100 \%$. $90 \%$ of respondents rated the $\mathrm{MBC}$ as good to excellent and would recommend this program to a friend

Conclusions: Online memory psychoeducation and support with community-dwelling seniors is feasible and positively received. Further development and evaluation of the effectiveness of these modules for support of diverse groups and those with a range of memory challenges appears warranted.

\section{being for Persons Living with Dementia in Rural Settings}

\section{Beverley Cassidy, Steven Wilton, Claire Checkland, Nick Zamora, Ashley Alders}

Background: Memory Cafes are social interventions for building inclusion and connection among community-dwelling adults living with dementia. Last year we piloted Nova Scotia's first Memory Cafes both in-person and then virtually during the COVID-19 pandemic in two rural communities (https://memorycafens.ca).

Methods: Winter and Spring Memory Cafe series were specifically designed to support the expressed needs of persons living with dementia as well as their caregivers/family members. Music and interactive art helped support the series' themes to promote cognitive stimulation, emotional expression and social connection in a supportive milieu. The narrative experiences of attendees were recorded and anonymous quality assurance questionnaires were given to assess self- reported changes in sense of well-being and social connectedness. Feasibility of running Memory Cafes was assessed through quality assurance feedback from multi-disciplinary Memory Café teams.

Results: $100 \%(17 / 17)$ of in-person Memory Café participants responded to an anonymous self-report questionnaire following the Winter 2020 series of Memory Cafes. $100 \%$ of participants reported improved sense of well-being pre-post series and $77 \%$ reported increased social connectedness. Narrative reports on participant experiences were highly positive regarding artist/musician involvement and for social connection with their family members and co-participants. Feedback from the 2021 Winter and Spring virtual series also will be presented.

Conclusions: Memory Cafes appear to be feasible and acceptable models with the potential to improve social connection and sense of well-being for persons with dementia and their caregivers. Adaptation of Memory Café models for other diverse rural settings including First Nations and Acadian communities would be important next steps. 


\section{Julia Kirkham, Alison Freeland, Chris Wilkes, Doug Urness}

Background: Choosing Wisely is an international, clinician led campaign to promote care that is supported by evidence, free from harm, and necessary. In 2015, the Canadian Psychiatric Association (CPA) partnered with the Canadian Academy of Geriatric Psychiatry and the Canadian Academy of Child and Adolescent Psychiatry to develop Choosing Wisely (CW) recommendations across the life span.

Methods: Ideas were generated from surveying each provincial psychiatric association, and $\mathrm{CW}$ recommendations were subsequently developed and vetted through the provincial associations with final approval from the boards of the 3 national associations. Since then, $\mathrm{CW}$ recommendations have been reviewed annually by the CPA Choosing Wisely working group, with modifications made to reflect updated evidence and feedback from stakeholders.

Results: The current CW statements will be presented, and recent changes to the original $\mathrm{CW}$ statements will be reviewed with a discussion of the rationale for the changes. Geriatric psychiatry focused $\mathrm{CW}$ statements from other jurisdictions will be presented. Special attention will be given to the importance of these recommendations for anxiety, depression and substance use treatment for older adults during the COVID pandemic. Current research and reports exploring the impact of $\mathrm{CW}$ on psychiatric practice will be reviewed will be discussed.

Conclusions: Participants will have an opportunity to bring potential topics of interest and provide feedback to CPA Choosing Wisely working group members regarding development of future $\mathrm{CW}$ geriatric psychiatry related statements.

\section{David Conn, Dr. Kiran Rabheru, Claire Checkland}

Background: Despite recent surveys that indicate that cannabis consumption among older adults has been accelerating at a much faster pace than other age groups, communication between older adults and healthcare providers often remains limited regarding cannabis. Cannabis use is not part of a standard assessment by most healthcare providers and older adults do not always report their cannabis use.

Methods: CCSMH assembled a Scientific Planning Committee including multi-disciplinary subject matter experts to create a series of e-learning modules. An anonymous needs assessment survey was carried out in the Fall of 2020. Over 1500 respondents (physicians, nurse practitioners, other healthcare providers, healthcare students, older adults and caregivers) responded to the survey. Development of the elearning modules was informed by the results of the needs assessment as well as other national surveys and scientific literature. Accredited e-learning modules will be launched in the Fall 2021 with accompanying KT resources to aid healthcare providers in providing optimal care to older adults.

Results: More older adults reported they are accessing information about cannabis from the internet (45\%) or friends/ family (34\%) than their physicians ( $40 \%) .41 \%$ of older adults that had spoken to their doctor about cannabis responded that their doctor was not able to answer their questions.

Conclusions: There is a gap in communication regarding cannabis and older adults. Healthcare providers and healthcare students reported they are eager to learn more about how to talk with patients, how to authorize and prescribe appropriately, how to mitigate risks and assess for cannabis use disorder in older adults.

\section{Behaviour Activation Tools \\ Keri-Leigh Cassidy, Kiran Rabheru, David Conn, Claire Checkland}

Background: The Brain Health and Wellness (BHW) Project was a national knowledge translation (KT) project led by the Canadian Coalition for Seniors' Mental Health (CCSMH). The objectives of the project were to assess the feasibility, acceptability, and effectiveness of wide scale-up of brain health promotion tools developed by the Fountain of Health $(\mathrm{FoH})$ in front line care in Canada.

Methods: The BHW Project nationally disseminated FoH behaviour change tools (a paper-based Health Behaviour Change Toolkit and web-based Wellness App) to clinicians in a wide variety of settings. Trained clinicians used $\mathrm{FoH}$ tools with patients to set a S.M.A.R.T. goal and offer a follow-up. Outcome measures included the impact of clinician education on translation into clinical practice, and on patients' self-reported health behaviours and attitude changes at onemonth follow up.

Results: Clinicians ( $\mathrm{N}=2184)$ from a variety of healthcare settings across Canada attended a knowledge transfer session. $35 \%(n=759)$ requested the Behaviour Change Toolkit to use in their practice. At one-month follow up, most patients (89\% of paper users and $90 \%$ of the Wellness App users) at least partially attained their health goal, and significantly improved self-rated wellbeing ( $85 \%$ of paper users and $80 \%$ of App users) and health attitudes.

Conclusions: The BHW Project demonstrated effectiveness of the CCSMH network for national scaling of KT of health behaviour change tools in Canada. The FoH tools, both paper-based tools and app, were found to be effective in activating patient health behaviours and in improving patient health attitudes.

\section{Associated with Treatments to Optimize Outcomes}

\section{Dallas Seitz}

Summary: This session will present new knowledge related to pharmacological management of older adults with latelife psychosis (LLP). The first presentation will provide an overview of existing randomized controlled trial evidence related to the use of antipsychotics in LLP. New evidence on 


\section{CAGP-CCSMH ANNUAL SCIENTIFIC MEETING: BOOK OF ABSTRACTS}

the patterns of antipsychotics use among older adults with LLP will be presented from a population-based real world study. This study will provide an overview of the characteristics of individuals with LLP including medical and psychiatric comorbidity. The patterns of antipsychotic use will be described including measures of polypharmacy and dose. The second session will then present evidence on the association between patterns of antipsychotic use and all-cause mortality among older adults with LLP. This study will describe how various prescribing practices may by associated with increased risk of mortality and other adverse events. The final presentation will provide an overview of an overview of an integrated care pathway for older adults with LLP and present evidence for the utility of care pathways for older adults with LLP. Attendees will learn new information related to the use of antipsychotics in LLP and identify strategies to optimize prescribing for this vulnerable and growing population.

\section{Based Use of Antipsychotics}

\section{Petal Abdool, Mulsant, Benoit H, Rajji, Tarek K, Patel Kinjal, Supasitthumrong Thitiporn}

Background: Antipsychotic use in older patients is associated with many adverse effects, including tardive dyskinesia and extrapyramidal symptoms, which, in turn, increase the risk of falls. Antipsychotics are also associated with metabolic syndrome and cognitive impairment in older patients. Integrated care pathways (ICPs) are designed to manage specific conditions using standardized assessments and measurementbased interventions. This study aims to compare the use of recommended tools to monitor for adverse effects associated with antipsychotics in older patients managed within an ICP and those managed under usual care conditions or treatment as usual (TAU).

Methods: We reviewed and compared the health records of 100 older patients enrolled in an ICP for late-life schizophrenia with those of 100 older patients treated with antipsychotics under TAU conditions.

Results: Monitoring rates were significantly higher in the ICP group than in the TAU group for all assessments: extrapyramidal symptoms ( $94 \%$ versus $5 \%$ ), metabolic disturbances (91\% versus $25 \%$ ), fall risk ( $82 \%$ versus $35 \%$ ), and cognitive impairment (72\% versus $28 \%$ ). Rates of antipsychotic polypharmacy were also six times higher in the TAU group.

Conclusions: Older patients with schizophrenia treated with antipsychotics within an ICP experience higher rates of monitoring and less psychotropic polypharmacy than older patients treated with antipsychotics under TAU conditions. These findings suggest that an ICP can improve the quality of antipsychotic pharmacotherapy in older patients and thus possibly its effectiveness. This needs to be confirmed by a randomized controlled trial.

\section{Dallas Seitz}

Background: There are increasing numbers of older adults with schizophrenia and other types of late-life psychosis (LLP) $>$ Antipsychotic medications are an important part of optimizing mental health outcomes and quality of life for this population. To date there have been relatively few randomized controlled trials or observational studies examining the patterns of antipsychotics in older adults with LLP.

Methods: We describe the patterns of antipsychotics use in older adults with LLP using population-based administrative databases in Ontario, Canada. We first identified all adults aged 66 years and older in Ontario, Canada who met criteria for LLP using two validated algorithms for psychotic disorders: a sensitive case definition and a specific case definition.

Results: Using these definitions a total of 33,672 individuals were included in the sensitive cohort and 4,481 individuals were included in the specific cohort. In the sensitive cohort, $55 \%$ of individuals received no antipsychotics in the preceding 120 days, while monotherapy with typical antipsychotics, atypical monotherapy and antipsychotic polypharmacy were received by $7.7 \%, 28.6 \%$ and $8.3 \%$, respectively, in the sensitive cohort. In the specific cohort $28.7 \%$ received no antipsychotics and typical monotherapy, atypical monotherapy and polypharmacy were received by $8.3 \%, 41.4 \%$ and $22 \%$, respectively. The mean dose of antipsychotics received were $11.2 \mathrm{mg}(\mathrm{SD}=14.4 \mathrm{mg}$ ) of olanzapine equivalents daily in the sensitive cohort and 17.5 $\mathrm{mg}(\mathrm{SD}=19.1 \mathrm{mg})$ in the specific cohort.

Conclusions: There is variation in the use of antipsychotics among older adults with LLP. Many older adults with LLP receive relatively high doses of medications and are prescribed medications in a manner that may place them at increased risk for adverse events.

\section{Wide Study}

\section{Soham Rej}

Background: Antipsychotic (AP) use in the elderly remains controversial in both non-dementia and dementia populations, although they are the main treatment for psychotic disorders. In Canada, there is a black box warning for their use in dementia for their potential for premature mortality. However, there is very little safety data examining whether or not APs are associated with mortality in late-life psychosis.

Methods: We conducted a province-wide cohort study. Patients were older adults aged $\geq 66$ between April 1st, 2008 and March 31st, 2016 from Ontario, Canada. Patients were included into a sensitive cohort $(\mathrm{n}=22,314$, any diagnosis of psychosis) or a specific cohort $(n=1,987$, hospitalization for psychosis). Patients were followed up to 5 years. Cox regression analyses compared adjusted hazard ratios (aHRs) for mortality in AP users and non-users, after controlling for important covariates that differed between groups (including health care/other medication use). 
Results: In the specific cohort, where $24.8 \%$ of the population was not using APs, atypical AP monotherapy was associated with reduced mortality in non-dementia (aHR 0.49 $(0.42-0.58), \mathrm{p}=0.004)$ and dementia (aHR $0.38(0.32-0.45)$, $\mathrm{p}<0.001)$ late- life psychosis populations. Similar results were observed in the sensitive cohort and with typical AP monotherapy and AP polypharmacy.

Conclusions: AP use appears to be independently associated with substantially reduced mortality in late-life psychosis.

\section{COVID-19}

\section{Sara Clemens, Carrie Heer, Audrey Devitt}

Background: The value of virtual care was tested as COVID-19 mounted its first wave across Ontario long-term care (LTC) and retirement homes. Many residents experienced reduced access to care. Emergency department visits, hospitalizations, and length of stay (LOS) were expected to increase, and quality of life was expected to plummet. Virtual care offered a solution to improve access and equity for residents in these homes.

Methods: Ten LTC and ten retirement homes were recruited for a 3-month Plan-Do-Study-Act. Participating homes received a free computer tablet, platform training, biweekly webinars, online toolkit resources and support from a nurse consultant and/or nurse practitioner. A project manager met weekly with each of the nurse consultants, conducted midpoint interviews with home leadership and multiple focus groups and interviews with residents, family members, staff and medical doctor (MD)/ NPs. Pre-post test surveys were conducted to determine home's technology needs. Nurse consultants conducted readiness assessments and compared these results to use-data and outcomes.

Results: Homes with the highest use of virtual care had lower emergency department transfers and hospitalizations than homes with lower use, and homes with the highest readiness scores had the highest use of virtual care. Physician engagement, home environment, and models for sustaining virtual care in the home impacted use of virtual care the most.

Conclusions: Homes should be encouraged to adapt virtual care when readiness factors are high to maximize related benefits to residents, staff and the community.

\section{Song Yang Yu, Aviva Rostas}

Background: Behavioural and psychological symptoms of dementia are some of the most common psychiatric symptoms for residents of long-term care facilities (LTCs). Psychiatric care for LTC residents is often provided by Geriatric Mental Health Outreach Teams (GMHOTs). Due to the COVID-19 pandemic, GMHOTs had to rapidly switch to a telemedicineonly model of care. This transition was accompanied by numerous challenges. It is important to develop an evidencebased model of telemental health delivery to LTCs. We aim to improve the quality of telemental health care provided by
GMHOTs, by developing a pre-visit checklist.

Methods: We reviewed current guidelines related to telemental health. We conducted stakeholder interviews, including experts on telemental health and CAMH GHMOT clinicians. We conducted an online survey of representatives from 21 LTCs in Toronto. We also documented the challenges encountered during telemental health assessments conducted by one CAMH GMHOT team over several months.

Results: Four interviews were conducted with experts on telemental health, and eight responses were received from LTC representatives. One common theme from the interviews and responses was the importance of accommodating the LTC residents' cognitive and functional limitations. It is important to have properly trained staff at the LTC who can facilitate the assessments. It is also essential to have properly functioning technology, as well as clear documentation of consent as part of the referral process for telemental health services.

Conclusions: Information from the interviews and surveys will be used to develop a pre-visit checklist to be used in the delivery of telemental health services to LTCs.

\section{Engagement for Older Adults (VCE Guide) Battles Geriatric Isolation}

\section{Simonne Cumberbatch, Melissa Tafler}

Background: Seniors across all sectors of care are experiencing significant decreases in meaningful social engagement due to COVID isolation. Social isolation is a determinant of health that results in serious health consequences e.g. risks of depression, alcoholism, and suicidal thoughts. In seniors, additional risks include autoimmune and neurocognitive problems, severe mental illness, increased incidence and worsening of dementia, and greater rate of mortality. Research indicates that meaningful social engagement is as important to seniors' health as maintaining personal hygiene. Racialized and low- income seniors face disproportionate risks of COVID contraction and isolation. As healthcare leaders, we should be paying attention to this.

Methods: To address this increase in under-stimulation, isolation and loneliness, The Ontario CLRI at Baycrest and TCLHIN Behavioural Supports Ontario developed and released "A Guide to Virtual Creative Engagement for Older Adults" (The VCE Guide). This guide helps users navigate the free online resources available to support therapeutic engagement.

Results: The VCE Guide is a curated list of services appropriate for seniors with various health conditions and levels of ability. It includes summaries of diverse services, how to access them, supportive recommendations, and guidance around assessing the fit between available resources and client capability. The guide can help: address isolation-induced depression, creating a sense of community and connection, and in the management of responsive behaviours triggered by isolation or under-stimulation. 
Conclusions: The VCE Guide helps to keep QOL at the forefront of senior health care, supports equity in virtual community engagement, and provides crucial connections to overcome loneliness during COVID.

\section{Dementia Care Research}

\section{Ngozi Faith Iroanyah, Dr. Saskia Sivananthan, Haridos Apostolides}

Background: Approximately 560,000 people in Canada live with dementia, yet research on the experiences of ethnoculturally diverse populations is largely unknown. These gaps in knowledge have resulted in inefficiencies in service and care provision for these communities. To address these gaps the Alzheimer Society of Canada (ASC) and the College of Family Physicians of Canada (CFPC) collaborated in the development of a national study to understand the experiences of persons living with dementia (PLWD) and caregivers, with healthcare providers.

Methods: This study used quantitative data collection methods through the design of 2 targeted surveys, one for family physicians and the other for PLWD and caregivers. A community engagement strategy was specially developed to ensure the inclusion of ethnocultural groups using a multi-tiered selection process. Descriptive statistics will be used to analyze the responses and thematic analysis will be used to code data and generate themes.

Results: This survey is the first of its kind in Canada to investigate the relationships between people living with dementia and caregivers, with their primary health providers. It will also bridge the knowledge gap in dementia care research by focusing on inclusion of ethnocultural communities. This focus has resulted in increased interest and uptake of the survey.

Conclusions: Findings from the survey will guide the ASCCFPC collaboration in the development of resources to better support physician management of patients with dementia. It will also guide ASC's development of new culturally diverse resources, research initiatives and educational materials for PLWD and caregivers, and help create and strengthen community relationships.

\section{Reviewers}

\section{Kaitlyn Jaggers, Jocelyn Badali, Josée Guimond, Saskia Sivananthan}

Background: Since 2014, the Alzheimer Society of Canada (ASC) has included people with lived experience of dementia (PWLE) in the Alzheimer Society Research Program's (ASRP) peer review process through the role of the Citizen Reviewer, however, this has been limited in scope. In 2019, a pilot study expanded this role to a broader panel of Citizen Reviewers as equal participants in the peer review process, scoring applications and providing valuable contextual feedback. In 2020, this process was further formalized, allowing for greater participation, inclusivity, and engagement.

Methods: Qualitative feedback was collected from panelists following the 2019 pilot which demonstrated a collective desire to improve overall engagement while formalizing a systematic process. The data was thematically assessed and used to create several resources to virtually recruit, maintain, and train Citizen Reviewers as equal and valued participants in the peer review process.

Results: Data from the 2019 pilot resulted in a four-step process for recruitment, orientation, and training of Citizen Reviewers. This process included semi-structured interviews, a comprehensive orientation package, a virtual orientation session, and technical support meetings. This, in turn, has led to the creation of a new resource guide for engaging PWLE in research, which demonstrates how to operationalize engaging and valuing the experiences of people affected by dementia.

Conclusions: The expansion of the Citizen Reviewer role has created a new way to improve the inclusion of those with lived experience within the evaluation of research proposals. While requiring significant preliminary preparations, the value of real-life experience in prioritizing research is unparalleled.

\section{Isabelle Martin-Zement, Marie-Isabelle Nadeau-Lessard}

Background: La maltraitance envers les aînés est un enjeu mondial connue depuis plusieurs années, mais davantage mise au premier plan dans le contexte de la COVID-19. Cette réalité serait en augmentation vue la croissance absolue de la population âgée.

Methods: Au Québec, la lutte à la maltraitance s'organise principalement autour des mesures du plan d'action gouvernemental pour contrer la maltraitance envers les personnes aînées. Une revue de ces mesures, de la littérature sur la maltraitance au Québec envers les aînés et des statistiques récentes sera effectuée. Les facteurs de risques et de vulnérabilité de l'aîné seront discutés.

Results: La maltraitance envers les aînés touche une grande partie de la population âgée et seulement la pointe de l'iceberg est visible. Deux formes et sept types de maltraitances sont reconnues, dont les types les plus fréquents sont la maltraitance psychologique et financière. Les femmes seraient plus souvent impliquées comme victime, et les proches seraient responsables dans une grande proportion des cas. Plusieurs facteurs nuisent à la recherche d'aide, dont le sentiment de honte ou afin de protéger la personne maltraitante. Le dépistage et les interventions doivent être personnalisées tout en étant empreinte d'inclusion et d'équité.

Conclusions: La maltraitance envers les aînés est un enjeu majeur et d'actualité auquel nous devons faire face individuellement et collectivement. Nous devons revoir notre façon d'accompagner et de soutenir les ainés dans leur processus de vieillissement. Nous devons aussi appliquer un dépistage aux aînés, surtout lorsque ceux-ci sont en situations de 
vulnérabilité. Identifier des situations où une personne âgée est victime de maltraitance est primordiale, tout en étant ouvert et sensibles aux valeurs de l'individu et de son bagage socio-culturel.

\section{Kelly Davies, Kim Schryburt-Brown}

Background: All people have a need to love and be loved by others, regardless of their age or living location. There is a gap in resources available to Long Term Care Homes (LTCHs) which help them meet residents' need for love and belonging while keeping them safe from abuse.

- LTCHs only have an abuse algorithm to guide their response to intimate acts between residents;

- Not every encounter between two residents is abuse.

Resources are needed to guide decision making around whether an intimate encounter involving residents of LTC is abuseor a need for love and belonging.

Methods: Stakeholders from a variety of sectors were invited to join a working group. Members represent people with lived experience, the LGBTQ2S community, LTCH staff, Seniors Mental Health staff, and specialized adult mental health staff. Group members use quality improvement tools to identify root causes and then developed a risk assessment as well as decision algorithms for verbal and physical interactions.

Results: This workshop will provide a brief overview of the theoretical constructs underlying this work, the risk assessment and decision algorithms developed for front line staff use. We will present the results of a PDSA cycle to detail how the risk assessment and algorithms work in real life, with outcome measures including PRN medication use, "sexually inappropriate" critical incidents and resident quality of life.

Conclusions: Participants will be guided through case scenarios to demonstrate how they can use the risk assessment and decision algorithms to promote love and belonging in residents of LTC.

\section{Older Adults Living with Complex Health Needs}

\section{Sophiya Benjamin}

Summary: To create equitable access to health services that meet the needs of our aging society, we must reimagine our siloed models of care. As we face an ongoing shortfall in the numbers of trained specialists in geriatric psychiatry, an integrated approach that leverages the diverse skills of health disciplines (e.g. nursing, medicine, physiotherapy, etc.) and sectors (e.g. primary, specialty, acute, community, long-term care, etc.) offers the promise of effective service delivery to address interrelated physical, mental and social health issues. To achieve this approach, we must first understand the design elements of integrated care relevant to the care of older persons living with complex and chronic health issues. We must also understand the numbers of older adults living with complexity/frailty using population level data to estimate the current need. Since its inception, Provincial Geriatrics Leadership Ontario (PGLO) has completed several important projects that can help inform the work of system leaders across Canada. In this symposium, we will present results from selected projects, which include 1) a scoping review on the components of integrated care 2) estimates of older adults living with complexity/frailty in Ontario and 3) practical examples of integrated, cross-sectoral responses to the COVID-19 pandemic to support vulnerable older adults and health care workers. This symposium is both a sharing of resources with the larger geriatric mental health community and a call to action for leaders in geriatric mental health to join in the co- design of emerging work related to the above presentations.

\section{Adam Morrison}

Background: Integrated care is an evidence-based approach for meeting the interrelated physical, mental, and social health needs of older adults, and for meeting targets in health quality and efficiency. This session highlights the results of a scoping review that Provincial Geriatrics Leadership Ontario conducted to identify design elements specific to integrated care for older adults living with complex and chronic health and social care needs.

Methods: We conducted a scoping review to identify the core design elements and infrastructures that come together to form integrated care for older persons living with complex and chronic health and social care needs. We used defined criteria to scope the literature for recent (i.e. 2017-2020) frameworks and practice models relevant to older adults. We then analyzed these documents to identify common patterns in the implementation of integrated care relevant to older adults living with complex and chronic health and social care needs.

Results: Our analysis uncovered 13 design elements underpinning integrated care for older persons, and ways these elements can be taken up in practice. We examined elements such as: multi-disciplinarity; cross-sector partnerships; comprehensive assessment and care planning; integrated specialized geriatric expertise; older person-centred care; engaged older persons and family/friend caregivers; and others.

Conclusions: This session provides suggestions for how to implement the designs elements in practice, resulting in better, more integrated care for older adults. We also discuss how these design elements shape our thinking as a provincial organization and inform the development of an indicator set that will measure integrated care for older adults in Ontario.

\section{Kelly Kay}

Background: Planners and clinicians alike need information about anticipated demand, along with information about current service availability and utilization to better respond to the needs of an older population. In this session, examples that compare anticipated service demand against current supply 
and utilization are provided, aligned with practice standards and other available benchmarks, to inform decision-making approaches about current and future health service design.

Methods: Based on the work of Hoover et al. (2013), Provincial Geriatrics Leadership Ontario has estimated the prevalence of frailty by census division and projected these estimates to 2040 to facilitate planning for health services for older adults living with complex and chronic health concerns in Ontario. We have also combined these estimates with practice guidelines and considered geographic and socio-economic variation across the province to develop scenarios to predict how much service may be required by older adults living with complex health conditions.

Results: This work illustrates the magnitude and nature of anticipated demand for a sampling of health services, such as dementia reassessment and ongoing care for older adults living with complex health concerns (frailty).

Conclusions: This session reviews our approach and assumptions and invites participants to add their experience to inform current planning assumptions and consider how estimates such as these may facilitate a better understanding of the need for services that can respond to the specific requirements of older persons.

\section{Kevin Young}

Background: Attention is needed to identify emerging supports required by community dwelling older adults and their caregivers as Ontario plans for the recovery phase of the pandemic. This session arises directly from the reported experiences of older adults, caregivers and clinicians who have identified ongoing and often deferred needs, with changing expectations brought about by new approaches to integrated care to address the impact of the COVID-19 pandemic on community dwelling older adults.

Methods: Drawing on case examples from a variety of sources, we share experiences in the care of community-dwelling older adults living with complex health concerns both during and beyond the COVID-19 pandemic.

Results: Through examples of integrated, cross-sectoral responses to emerging needs, we identify major themes and practical supports for older persons' care, including tools and resources helpful to health care workers engaged in the care of community-dwelling older adults. We also examine how collaborative initiatives conceived during the pandemic can help leverage systemic change and a better patient experience.

Conclusions: This session highlights how the planning and delivery of equitable and accessible health services that move beyond silos to truly integrated, person-centred care can be achieved by leveraging clinical leadership in specialized geriatric services and others to co-design an array of supports that comprehensively meet the needs of the patient.

\section{Neuropathology of Late Life Depression: Clinical Predictors of Amyloid and Tau Neuropathology in Non-Demented Older Adults With Depression}

\author{
Doyoung Kim, Alex Kiss, Susan E. Bronskill, \\ Krista L. Lanctot, Nathan Herrmann, Damien Gallagher
}

Background: Previous studies regarding the relationship of depression with amyloid and tau neuropathology have reported conflicting findings. We examined whether depression is associated with higher Alzheimer's disease neuropathologic changes and whether sex moderates their relationships.

Methods: Using the National Alzheimer's Coordinating Center database (2005-2020), we conducted a cross-sectional study of non- demented older adults (age $50+$; CDR $\leq 0.5$ ) who had autopsy within 1 year of their last clinic visit. Regression models were fitted to determine if recent and/or remote depression were independently associated with amyloid spread measured via modified Thal phase scoring for amyloid beta deposition, tau measured using modified Braak stage for neurofibrillary degeneration, and density of neuritic plaques measured with CERAD score. We also determined if any of their associations were moderated by sex.

Results: We included 395 participants (94 Thal, 393 Braak, and 394 CERAD). Those who had recent (within previous 2 years) but not a remote history of depression were more likely to have higher Thal phase compared to those without a history of depression (Odds Ratio $=13.3 ; 95 \%$ CI, 1.5-116.3). Sex did not moderate the association between recent depression and Thal phase. No significant associations between depression and Braak or CERAD scores were observed.

Conclusions: Our findings indicate that the association between late-life depression and Alzheimer's neuropathology is explained by spread of amyloid pathology beyond cortical regions to include subcortical regions rather than density of neuritic plaques or neurofibrillary tangles.

\section{Association with Clinical Symptoms: A Systematic Review and Meta-analyses}

\section{Sanjeev Kumar, Shaylyn Joseph, Rachel Patterson, Wei Wang, Daniel Blumberger, Tarek Rajji}

Background: Alzheimer's Dementia (AD) is characterized by cognitive and neuropsychiatric symptoms (NPS) due to underlying neurodegenerative pathology. Studies using electroencephalography (EEG) have shown increased epileptiform and epileptic activity in AD. This review and meta-analyses aims to synthesize the existing evidence for quantitative abnormalities of cortical excitability in $\mathrm{AD}$ and their relationship with clinical symptoms.

Methods: We systematically searched and reviewed publications that quantitatively assessed cortical excitability, using transcranial magnetic stimulation (TMS) resting motor threshold (rMT), active motor threshold (aMT), motor evoked 
potential (MEP) or directly from the cortex using TMS-EEG via TMS-evoked potential (TEP). We meta-analyzed studies that assessed rMT and aMT using random effects model.

Results: We identified 895 publications out of which 37 were included in the qualitative review and 30 studies using rMT or aMT were included in the meta-analyses. The AD group had reduced rMT (Hedges' $\mathrm{g}=-0.99,95 \%$ CI [-1.29, -0.68], $\mathrm{p}<$ 0.00001 ) and aMT (Hedges' $\mathrm{g}=-0.87,95 \% \mathrm{CI}[-1.50,-0.24]$, $\mathrm{p}<0.00001$ ) as compared with control groups, indicative of higher cortical excitability. Qualitative review found evidence of increased MEP amplitude, whereas findings related to TEP were inconsistent. There was some evidence supporting an inverse association between cortical excitability and global cognition. No publications reported on the relationship between cortical excitability and NPS.

Conclusions: There is strong evidence of increased motor cortex excitability in $\mathrm{AD}$ and some evidence of an inverse association between excitability and cognition. Future studies should assess cortical excitability from non-motor areas and examine its relationship with cognition and NPS.

\section{Autopsy Register in a Community Sample of 1,143 Older Adults}

\section{Paula Villela Nunes, Atmis Medeiros Haidar, Livia Mancine, Beatriz Astolfi Neves, Renata Elaine Paraizo Leite, Carlos Augusto Pasqualucci, Beny Lafer, Rogerio Salvini, Claudia Kimie Suemoto}

Background: Depression is associated with high mortality. Its etiology is multifactorial and still can be better clarified. There are few studies on the causes of death $(\mathrm{CoD})$ using autopsy registers and Major Depressive Disorder (MDD) diagnosis according to the SCID. This study aims to compare cardiovascular causes of death in individuals with and without MDD in a community sample.

Methods: Participants were deceased subjects submitted to autopsy in community-based service. Inclusion criteria were age 50 years or older, non-traumatic $\mathrm{CoD}$, and a knowledgeable informant. Lifetime MDD was defined as at least one previous episode according to the Structured Clinical Interview for DSM (SCID). We included 1,143 participants from the Biobank for Aging Studies of the University of São Paulo (mean age $=70.6 \pm 11.7,54.8 \%$ male). We used multivariable logistic regression models in the analysis, adjusted for age, gender, race, and schooling.

Results: MDD was found in 243 cases (21.3\%). Cardiovascular was the most common $\mathrm{CoD}$ in the sample $(\mathrm{n}=790$; $69.1 \%$ ). MDD was not associated to greater cardiovascular $\mathrm{CoD}(\mathrm{p}=0.900)$. Similar results were found when considering specific CoD such as myocardial infarction $(\mathrm{p}=606)$, cardiomyopathy $(\mathrm{p}=0.271)$ hypertensive heart disease $(\mathrm{p}=0.105)$, heart failure $(\mathrm{p}=0.783)$, and aortic aneurysm $(\mathrm{p}=0.936)$. When using information of comorbidity that was also present in the autopsy registers no association was found.
Conclusions: Cardiovascular CoD were not associated to MDD in this large community sample, even after correcting for differences in gender, amongst others. Future studies addressing other variables and other CoD should be granted.

\section{Annalee King, Dr. Geoff Daniel, Nancy Hooper, Sandra Easson-Bruno, Tamara Nowak Lennard}

Background: Specialized Geriatric Services in North Simcoe Muskoka, had a vision within the clinical design to create a single system of care through the integration of the Geriatric Psychiatry Consultation service and Behaviour Support Services. For years, community stakeholders completed multiple referrals to access specialized services across the region resulting in lengthy wait times and frustration for patients, families and service providers. Both teams supported many clients, yet they worked in isolation of each other.

Methods: In early 2020, the design came to fruition with the goal of creating a single integrated system of care. Implementation of mental health rounds to support inter-professional collaboration \& education. Clinician shadowing, peer mentoring, capacity building and participation in workflow mapping with all team members to support building relationships and fostering change management approaches. Capitalized on ubiquitous virtual platforms to connect the team.

Results: Integration to create a single team has proved to be both innovative and efficient. Increased satisfaction from referral sources and the average wait time for psychiatrist consult improved by 55\%. Evidence of improved team confidence, staff satisfaction and engagement in practice change as a result of inter-professional collaboration. Increased satisfaction with reduced duplication for clients, families and care providers to repeat their story to multiple service providers.

Conclusions: This successful integration demonstrates improved client-centered outcomes, increased system access and equity to psychiatry consultation, clinician growth and development and increased satisfaction all around.

\section{Community of Practice in Champlain}

\section{Martina Greco, Martina Greco, Sabrina Veri}

Background: The Behaviour Supports Ontario (BSO) initiative was developed to enhance health care services for older adults with complex responsive behaviours associated with dementia, mental health, substance use and/or other neurological conditions. BSO is a collaborative care model, BSO Champions are embedded roles in Long-term Care Homes (LTCHs) and work in collaboration with Outreach team members to conduct behavioural assessments, execute care plans and strategic behavioural interventions with residents. In collaboration with the BSO Outreach Team, BSO Champions are responsible for building capacity with their staff in home. A Community of Practice (CoP) was created to support and facilitate knowledge translation through a network of BSO Champions across the Champlain area. 
Methods: Logic Model Framework, Virtual monthly meetings, Email .

Results: Previously this group of individuals met annually. The CoP enables BSO Champions from LTCH's across the Champlain region to participate in a monthly peer-supported learning workshop, which in turn enhances their skillset, enabling them to improve patient care. The CoP development will identify, and inform future needs regarding education, training, $\mathrm{BSO}$ annual conferences and act as a platform for standardized practice dissemination in alignment with BSO PCO.

Conclusions: The goal of this $\mathrm{CoP}$ is to focus on capacity building amongst BSO Champions across the Champlain area by providing collaboration through peer-supported learning, resource sharing, education, and networking and opportunities to discuss challenging tasks related to their roles. By improving skillset of BSO Champions it will result in an enhanced level of person centered care for the residents and their families they support in LTCH.

\section{Health Services for Older Adults in Canada to Practice and Policy}

\section{Alexa Bol, Laura Mullaly, Caroline Ostrom}

Background: Applying the principles and values found in the Mental Health Commission of Canada's (MHCC) Guidelines for Comprehensive Mental Health Services for Older Adults in Canada can ensure that your policy, program, or service is informed by and reflects the core values of Canadian older adults, as well as the system values that promote and support their mental health. These principles and values are based on the lived experience of diverse older adults and their families, as well as from evidence, literature reviews, and from consultations with key stakeholders from across Canada.

Methods: The Guidelines for Comprehensive Mental Health Services for Older Adults in Canada provide evidence-based recommendations to service providers and policy makers on ensuring a comprehensive service system that is inclusive of the mental health of older adults in a variety of settings.

Results: The original guidelines as well as additional, related KT products are available on the MHCC's website (https:// www.mentalhealthcommission.ca/English/what-we-do/ seniors). There are additional supporting documents and checklists related to applying the Guidelines in a Covid context as well as applying the principles and values to services and policy.

Conclusions: Our current tools and resources will be presented and shared during this workshop. These tools aim to move the evidence-based ideas presented in the Guidelines for Comprehensive Mental Health Services for Older Adults in Canada into practice on a broad scope across Canada. Further tools and resources are being created to continue to support policy makers and service providers in this implementation process.

\section{WITHDRAWN}

\section{"It was like living in a prison" Loneliness in Independent-Living During COVID-19}

\author{
Dan Huynh, Alice Kong, Dr. Lilian Thorpe
}

Background: Loneliness is a subjective experience that can lead to serious psychological and physical consequences, especially in older adults. Unfortunately, rates of loneliness among Canadian seniors have dramatically increased since the onset of COVID-19. Particular attention from mainstream media has been directed towards seniors living in long-term care settings due to severe social distancing restrictions. However, an excluded and vulnerable group of seniors also suffering from loneliness are those living in retirement and independentliving homes, which have been exposed to restrictions similar to those in long-term care. As such, a knowledge gap exists in understanding loneliness during COVID-19 in this population as they could have been subjected to a significant departure from their normal lifeworld. The objective of our study is to uncover the meaning of loneliness for older adults living in independent-living homes during the pandemic.

Methods: From April 2021 to August 2021, ten to twenty-five residents from an independent living home in Saskatchewan will be purposefully selected to participate in semi-structured interviews exploring their lived experiences throughout the pandemic with a focus on loneliness. Interpretive phenomenological analysis will be used to systematically analyze interview recordings and transcriptions.

Results: Themes and concepts related to loneliness that contribute to how participants make sense of their personal and social world during the pandemic will be reported.

Conclusions: The knowledge obtained through our study will allow healthcare providers to take steps towards empathizing with these individuals experiencing loneliness during the pandemic which will further foster therapeutic alliances and create future interventions.

\section{WITHDRAWN}

Asynchronous e-learning as Part of a Blended Teaching Strategy to Enhance Psychiatry Resident Knowledge of ECT: Design, Development, Implementation and Evaluation

\section{Sarah Payne, Karen Saperson, Michael Brown, Anthony Levinson}

Background: Prescribing electroconvulsive therapy (ECT) is now considered a core Entrustable Professional Activity within the Canadian geriatric psychiatry training program. When gaps in learning in prescribing of ECT for older adults were identified in the formal curriculum for psychiatry 
residents at McMaster University, an e-learning course was developed to address this educational gap using a blended learning curriculum, with asynchronous e-learning complementing experiential activities.

Methods: Key learning objectives were developed and the e-learning course created using best practices in multimedia learning; it was designed to focus on case-based questions without relying on didactic delivery of content. The course consisted of prerequisite readings, two case-based modules, a post-test, and evaluation survey. The course was deployed through the Medportal learning management system and piloted in February 2018 with PGY2/PGY3 residents in geriatric psychiatry rotations. Using learner feedback, it was subsequently embedded within the formal curriculum.

Results: Twenty-one learners completed the course from February 2018-February 2021, with a median time to completion of approximately one hour. In the evaluation, $100 \%$ of learners identified the course to be a valuable learning experience, one that they would recommend to their colleagues, and one that helped them to identify areas for further learning.

Conclusions: This highly reviewed e-learning course on ECT has been incorporated into the core curriculum in both general psychiatry and geriatric psychiatry training programs thereby advancing achievement of competence. This e-learning initiative may serve as a model for blended learning that can be used for other core curriculum components to support Competency Based Medical Education.

\section{Design, Development, and Implementation of a Series of Asynchronous Online Courses to Complement Competency-Based Postgraduate Training of Geriatric Psychiatry Sub-Specialty Residents}

\section{Anthony Levinson, Sarah Payne, Ana Hategan, Mandy Esliger, Kathleen Singh, Catherine Hickey, Terry Chisholm}

Background: High-quality, online resources can effectively complement experiential learning; but it can be challenging for a single institution to develop a comprehensive series of online courses. Our objective was to collaborate on the development and implementation of asynchronous e-learning to complement competency-based training in geriatric psychiatry.

Methods: Previously developed e-modules from Dalhousie and McMaster were aggregated within the same learning management system to facilitate a single point of access and standardize evaluation surveys. A consortium of interested institutions was formed and instructional design guidelines were developed in an attempt to achieve consistency with respect to the design and development of modules, as well as alignment with best practices in evidence-based e-learning. Courses were mapped to the Competence by Design stages of training and Entrustable Professional Activities.
Results: Currently, there are 11 courses within the learning path covering key topics such as: Approach to the Geriatric Psychiatry Assessment; Geriatric Depression; Late-Life Psychosis; and others. Residents from 5 programs have enrolled. Post-course evaluations show a high degree of satisfaction with the courses. Any sub-specialty resident or program director across the country may request access to the learning path.

Conclusions: A roadmap has been created for additional courses, and several are in development. New institutions are contributing, and there will be efforts to engage programs across the country. There is also the potential to scale and spread the learning path to additional audiences, such as other residents and inter-professional team members working in seniors' mental health.

\section{Establishing a National ECHO Care of the Elderly: Mental Health Program}

\section{Lisa Sokoloff, Claire Checkland, Jasmeen Guraya, David Conn, Kiran Rabheru, Dallas Seitz, Sid Feldman, Vivian Ewa, Andrea Hunter}

Background: Project ECHO is a virtual, case-based capacitybuilding education program for healthcare providers. Baycrest, Canadian Coalition for Seniors' Mental Health and the Canadian Academy of Geriatric Psychiatry collaborated to launch a national ECHO for mental health and aging. This partnership, coordinated by a cross-Canadian Steering Group, allows for broad reach, including registration of learning partners from almost all Canadian provinces and territories. The program was funded by the RBC Foundation.

Methods: ECHO COE: Mental Health pilot consisted of 2 cycles:

- 6 weeks focused on broader mental health topics (e.g., delirium)

- 10 weeks with more specific topics (e.g., substance use disorders)

Needs assessments of healthcare providers and older adults informed the program curricula. Evaluation included weekly satisfaction surveys, and pre and post evaluations.

Results:

Participants:

- 154 healthcare providers participated in the 6-week session - 9 provinces, 1 territory represented

Preliminary Findings (weekly satisfaction surveys):

- high overall satisfaction

- self-reported enhanced knowledge

- self-reported that program will enhance clinical practice

- almost all would recommend the program to others

Conclusions: A national ECHO COE: Mental Health program is an effective way to bring together clinicians who work with 
and are interested in the mental health and wellbeing of older adults for education sessions, collaborative and mutual learning as well as for cross-jurisdictional knowledge transfer. Collaborative, cross-professional learning supports the exchange of best practice in mental health for older adults, supports the development of collegial pan-Canadian professional support and can address health system inequities.

\section{The Knowledge Gap: Cannabis and Older Adults Needs Assessment}

\section{David Conn, Dr. Kiran Rabheru, Claire Checkland}

Background: Cannabis use and older adults has received little attention in the scientific literature and clinical practice despite recent survey results that indicate cannabis consumption among older adults has been accelerating at a much faster pace than it has among other age groups. CCSMH distributed a needs assessment survey to determine current knowledge, confidence and awareness of cannabis use and older adults in order to inform the development of e-learning modules.

Methods: CCSMH received over 1500 responses to an anonymous needs assessment survey to gather information on cannabis use and older adults in the Fall of 2020. Target audiences included physicians, nurse practitioners, other healthcare providers, healthcare students, older adults and caregivers to older adults.

Results: Results indicated that, although $89 \%$ of physicians and nurse practitioners are aware of older patients in their practice using cannabis, only $39 \%$ agreed or strongly agreed that they have sufficient knowledge and expertise to address older patients' and their caregivers' questions about cannabis. $37 \%$ of older adults using cannabis indicated that they are using cannabis without a prescription or medical authorization from a physician / nurse practitioner for medical /therapeutic reasons and $25 \%$ reported using cannabis for non-medical reasons. The most common reasons for cannabis use were reported to be related to pain, sleep and anxiety.

Conclusions: The needs assessment results provide a current reflection of cannabis use by older adults in Canada while also providing insight into a reported gap in current knowledge of healthcare providers.

\section{New Technologies in Dementia Care}

\section{Stephen Lee-Cheong, Shabbir Amanullah}

Background: Dementia is characterized by global cognitive dysfunction, which can manifest itself in a myriad of problems. These problems result in various difficulties for patients when it comes to performing their Activities of Daily Living (ADLs) and Instrumental Activities of Daily Living (IADLs), leaving patients who do not have the proper support extremely vulnerable. Technology has advanced over the past years allowing for the commercial use of GPS, introduction of 'smart phones' and 'smart home systems', as well as, the advancement of artificial intelligence. Through these new advancements it has been possible to develop new technologies designed specifically to help care for those with dementia. Through this, greater health equity and quality of life can be achieved, as currently the financial costs of human support are too great for many patients and their families to afford.

Methods: A literature search using PubMed was undertaken using the keywords of "dementia", "technology", and "management" that revealed 571 results. 147 were included in the review.

Results: There are many new technologies available to help manage dementia. Some are designed for patients to use and others are designed for caregivers to use. An example includes monitoring systems, which may be used in place of physical restraints and allow for more patients to be cared for at home.

Conclusions: New technologies in dementia care may improve the quality of life for patients and improve health equity. However, there are some questions surrounding their ease of implementation and the ethics surrounding them that require further discussion.

\section{Promoting Seniors Mental Health: A Vision for Suicide Risk Assessment and Management}

\section{Ann Jarvie, Amy Van Berkum, Shauna Graf}

Background: Those 65 years and older are amongst the highest risk for suicide and represent $18 \%$ of the population in Ontario's London-Middlesex Region. St. Joseph's Health Care London (SJHC) provides inpatient and ambulatory health services to seniors via an internal referral process from the Geriatric Ambulatory Access Team (GAAT) which triages up to 3600 referrals per year. SJHC London is also the first Canadian organization to adopt the Zero Suicide Framework, focused on enhancing suicide prevention. Beginning in March 2021, the GAAT program and Zero Suicide team implemented new suicide prevention tools and processes which include all seniors being screened for suicide risk and policies and procedures which outline the appropriate suicide risk level interventions.

Methods: The Zero Suicide team, collaborating with clinicians in the mental health and GAAT programs, completed a review of best practices focused on suicide risk assessment and management algorithms to develop current processes. Mixed methods data collection via the Electronic Health Record and written clinician anecdotes will provide pertinent patient demographics, screening rates, risk levels, interventions, and self-reported perceptions of clinician competency. Rates of service collaboration for at risk patients with the local senior crisis team will also be reported.

Results: Evaluation is currently underway, however anticipated findings suggest there will be improved rates of suicide screening and safety processes for supporting high risk clients during transitions in care. Further, we suspect that clinicians 
will have clarity related to suicide risk processes and report improved levels of interdisciplinary communication of suicide risk status and competency with suicide management.

Conclusions: Implementing evidence-based processes that focus on suicide prevention for seniors have multiple benefits. Not only is an often-overlooked population screened and managed based on suicide risk, improved clinician competency and collaboration with other service collaborators enhances safety during high-risk transitions of care.

\section{WITHDRAWN}

\section{Systematic Review of Dementia Screening in the Arab World}

Reham Mansour, Dr Shabbir Amanallah

Background: Dementia is a syndrome in which there is deterioration in memory, thinking, behaviour and the ability to perform everyday activities. Although dementia mainly affects older people, it is not a normal part of aging. Worldwide, around 50 million people have dementia, and there are nearly 10 million people new cases every year, Alzheimer's disease is the most common form of dementia and may contribute to $60-70 \%$ of cases. ${ }^{(1)}$ In Canada the number of people living with dementia continue to rise, the increase is due to growth in Canada's seniors population, which is expected to rise $68 \%$ over the next 20 years, ${ }^{(2)}$ uncertainty of what tool to be used; makes screening and diagnosis of dementia underestimated in some areas due to challenges like: time, resources, awareness and lack of training on the use of the appropriate scale.

References: WHO. "Key facts.” Last modified September 21,2020. www.who.in; Canadian institute of health information. www.cihi.ca

1. Methods: To review the literature for the last 20 years using MEDLINE and PsycINF databases. The inclusion criteria are: 1- Screening was done in Arabic countries.

2. The screening results were published in a good journal.

3. The used tool should be one of the standardized tests, MoCA or MMSE.

Results: We will check how using different scales influence the results of the screening.

Conclusions: The use of different scales for screening and follow up may result in different outcomes based on the tool originally used for the diagnosis.

\section{Cognitive Remediation Therapy for Participants with Late-Life Schizophrenia}

\section{Angela C. Golas, Bishoy M. Elgallab, Petal S. Abdool, Christopher R. Bowie, Tarek K. Rajji}

Background: Cognitive deficits strongly predict function in schizophrenia. Anticholinergic medication burden (ACB) compounds age- related cognitive declines in late-life schizophrenia (LLS). Cognitive remediation (CR) improves cognition in schizophrenia, however literature in LLS is sparse. This study examined the effect of CR on cognitive performance in participants with LLS. We also assessed CR tolerability, the interaction between ACB and the effect of CR on cognition.

Methods: We adapted CR from our pilot study to a larger group. CR was provided over 12, twice-weekly, therapistguided group sessions. Computerized drill-and-practice exercise difficulty levels were adjusted automatically based on performance. Participants were assessed at baseline and at study completion using clinical and cognitive measures.

Results: Thirty-four participants were enrolled, 20 (mean (SD) age: 66.0 [5.8]) completed $92.3 \%$ (22.14 [1.98]) CR sessions; the ITT group $(\mathrm{N}=34)$ attended $72.9 \%$ (17.50 [7.23]) sessions. Global cognition did not improve (completers: $p=80$, ITT: $\mathrm{p}=0.86$ ) with negligible effect size (completers: $\mathrm{d}=0.08$; ITT $\mathrm{d}=0.04)$. There was no significant improvement in executive function (completers: $p=0.16$; ITT: $p=0.33$ ). Medication ACB was inversely associated with total MoCA score, accounting for $8.9 \%$ of the variance in final MoCA score $(\mathrm{p}=0.02)$.

Conclusions: Overall, CR was well tolerated but did not improve global cognition or executive functioning in this outpatient sample with LLS. A higher ACB was associated with greater cognitive impairment and a poorer response to $\mathrm{CR}$. Future studies need to better characterize potential variables serving to limit the response to $\mathrm{CR}$, including the number and frequency of CR sessions and the role of ACB.

\section{WITHDRAWN}

\section{"I Just Can't Take It Anymore": Using Suicide Notes to Understand Suicidality in Older Adults}

\section{Ari Cuperfain, Zainab Furqan, Mark Sinyor, Kenneth Shulman, Juveria Zaheer}

Background: Suicide is a complex multifactorial process that can result from a variety of biological, psychosocial and social stressors. The relative contributions of these stressors vary across the lifespan, as has been shown through epidemiological studies. Older adults face a unique set of challenges which may predispose them to suicidal ideation and suicide completion. Our study explored the subjective experience of mental illness and suicidality through the analysis of suicide notes, specifically in the older adult population.

Methods: Suicide note samples were obtained from the Office of the Chief Coroner for Ontario for suicide deaths in Toronto, Canada between 2003 and 2009. We employed a constructivist grounded theory informed analysis of written samples, whereby thematic analysis was conducted through line-by-line open coding, axial coding and theorizing of data. Demographic data and method of suicide were also collected. 
Results: A total of 29 suicide notes (average 221 words per letter; range 6-1095) written by individuals 65 years and older (average age 76.2 \pm 8.3 ) at time of death were included in the analysis. Expressed reasons for suicide centred on themes of burdensomeness, experiences of mental illness, loneliness/ isolation and physical health/disability. Suicide notes also elucidated the writers' conceptions of suicide as well as their emotional responses to stressors.

Conclusions: Suicide notes offer a unique window into the lived experience of those at highest risk of suicide. Our study can help bridge the gap between the known risk factors for suicide in older adults, with the subjective thoughts and emotions of those in distress.

\section{A Comparison of Placebo Effect in Two Randomized Controlled Trials of Pharmacotherapy or Neurostimulation in Treatment-Resistant Depression in Late Life}

\section{Rafae Wathra, Benoit Mulsant, Charles Reynolds III, Eric Lenze, Jordan Karp, Zafiris Daskalakis, Daniel Blumberger}

Background: Emerging research suggests the magnitude of the placebo effect differ depending on the intervention to which it is compared and the corresponding modality used as a "placebo". This differential placebo effect can have important implications in designing and interpreting randomized clinical trials (RCTs). There are no existing studies that have compared the effects of placebo in neurostimulation or pharmacotherapy RCTs in late-life depression.

Methods: Data was analysed from two RCTs of adults age 60 and older with a current treatment-resistant major depressive episode, one comparing aripiprazole and placebo pills and the other comparing repetitive Transcranial Magnetic Stimulation (rTMS) and sham rTMS. In both RCTs, depression was assessed with the 17-item Hamilton Depression Rating Scale (HDRS- 17). An analysis of covariance (ANCOVA) compared changes in HDRS-17 scores after 4 weeks in participants who received placebo pills or sham rTMS. Relevant covariates included years of education, duration of depressive episode, and baseline HDRS-17 score.

Results: Accounting for covariates, there was a larger reduction of HDRS-17 after 4 weeks in the sham rTMS group (estimated marginal mean + standard error: $-5.90+1.45$; $95 \%$ CI: $[-8.82,2.98])$ than in the placebo pills group $(-1.07$ $+1.45 ;[-3.98,1.85])$.

Conclusions: Sham rTMS may have a larger placebo effect than placebo pills early in the treatment of older adults with treatment- resistant depression. Differential placebo effects should be considered when interpreting results of RCTs or designing new ones.

\section{WITHDRAWN}

\section{The Fountain of Health for Optimal Aging: Results from a "5-Minute CBT" Online Course with Clinicians in Nova Scotia}

\author{
Amy Gough, Dr. Keri-Leigh Cassidy, \\ Dr. Michael Vallis, Ms. Jean Robinson-Dexter
}

Background: The Fountain of Health (FoH) is a national health promotion knowledge translation (KT) initiative using cognitive- behavioural therapy tools to activate behaviour change in key modifiable areas to promote health and resilience. A CPD- accredited online CBT course was developed for clinicians to use to activate their own self-care behaviours.

Methods: Clinicians enrolled in a 5-week accredited online CBT course offering KT on self-care using behavioural activation tools and S.M.A.R.T. (specific, measurable, actionoriented, realistic, time-limited) goal-setting. KT quality assurance outcome measures include: 1) pre-/post- clinician self-report on health attitudes and behaviours, and professional satisfaction, and 2) clinician goal-attainment scaling to assess behaviour change. Secondary outcome measures include correlations between "SMART-ness" of goals with goal attainment and clinician professional satisfaction.

Results: Results from 55 clinicians (physicians and allied health care professionals) enrolled in the Spring 2021 course will be presented.

Conclusions: We hypothesize the FoH online CBT-based course will positively impact clinician self-care attitudes and behaviours, and clinician professional satisfaction. Results will be used to guide future adaptation of the online course to reduce burnout and promote wellbeing.

\section{Suicidal Ideation Amongst Elderly Population}

\section{Ashitija Jasrai, Shabbir Amanullah}

Background: Suicidal ideation (SI) is a broad term used to describe a range of behaviors that involve contemplations and wishes of death, self-harm, and suicide. According to WHO, around 800,000 people suicide annually and it's been shown that suicide rates increase with age, with their highest levels being amongst the elderly population. This meta-analysis aims to synthesize extant literature that reports on suicidal ideation in the elderly population (65+ in age).

Methods: The systematic search will be done using multiple databases like PubMed, Scopus, MedLine (OVID), and PsychINFO following the Preferred Reporting Items for Systematic Reviews and Meta-Analyses (PRISMA) guidelines. Keywords used to find the literature will include "elderly", "older adults", "geriatric", "suicide", "suicidal ideation", and "self-harm". Only English primary articles will be selected based on whether they assess any form of suicidal outcome 


\section{CAGP-CCSMH ANNUAL SCIENTIFIC MEETING: BOOK OF ABSTRACTS}

amongst older adults along with usage of assessment tools like Suicidal Ideation Questionnaire (SIQ), Beck Scale for Suicide Ideation (BSSI), etc.

Results: Considering the current situation of increasing population, more older adults living alone, higher levels of stress and depression, along with increased loneliness due to the onset of the pandemic, the results are predicted to show an unfortunate increase in suicidal ideation amongst older adults.

Conclusions: This review will provide insight on suicidal ideation amongst older adults and help see where we lack, whether it be assessment tools, treatments, or prevention methods. This will help us direct future research towards resources for fixing areas we currently fall short in.

\section{Tunnel Vision: A Novel Investigation of the Effect of Depression on Field of View}

\section{Lisa Bolshin, Nasreen Khatri, Jennifer Ryan}

Background: This study examined the effects of depression on field of view and memory as it relates to cognitive remittance of depression. Depressed individuals possess a negativity bias producing a narrowed field of view, attentional bias, and enhanced memory for negative emotional information. The central conceptual question investigated whether formerly depressed (remitted) individuals are truly cognitively remitted and display the same emotional and attention inhibitions and memory as depressed individuals, specifically under a negatively mood induced state. None of these concepts have yet to be addressed together using eye tracking to study cognitive remittance.

Methods: Female participants aged 39-85 were grouped into non-depressed (healthy) and previously depressed (remitted) categories via diagnostic interview and subjective questionnaires. The study followed a 3-day testing procedure which included the diagnostic verification on the first day, and eyetracking methods on the second and third day. On the second day of testing participants saw a series of scenes and were asked to rate their emotional valence, and later recognize the scenes after a delay. After one week, participants returned for a third testing session which followed the same procedure with new stimuli. Neutral and negative mood inductions were randomized to either the second or third testing session.

Results: The study employed a $2 \times 3 \times 2$ mixed model ANOVA with three dependent variables (field of view, encoding, and recognition) and two independent variables (participant group and day/mood induction). Results supported the first hypothesis that suggested that formerly depressed individuals possess a narrower field of view, especially when subjected to a negative mood induction. The second hypothesis was also supported suggesting that a negative mood induction would result in greater visual exploration (attention) to negatively valenced information for formerly depressed individuals. Results failed to support the third hypothesis regarding recognition accuracy, yet formerly depressed individuals continued to demonstrate significantly different viewing patterns (i.e., saccade amplitudes and number of fixations) to emotionally valenced information under a negative mood induction.

Conclusions: Results from the current research illustrated that individuals who are remitted from depression do in fact process information in the environment differently than someone who has never been depressed. More specifically, they focus more on a narrow aspect of the environment, typically negative information, which negatively impacts their ability to efficiently encode information. Taken together, the results of the study support the idea that formerly depressed individuals experience a perceptual and cognitive tunnel vision that elaborates on their inhibited negative cognitive schemas and puts them at risk for relapse into further episodes of depression. 